\title{
Inaugural Message from the Editor-in-Chief
}

\section{George Perry}

It is with profound pleasure, humility and anticipation that we celebrate the launch of Journal of Systems and Integrative Neuroscience (JSIN) with this inaugural issue. On behalf of the JSIN Editorial Team, I would like to extend a very warm welcome to the readership of JSIN. I take this opportunity to thank our authors, editors and anonymous reviewers, all of whom have volunteered to contribute to the success of the journal.

Journal of Systems and Integrative Neuroscience (JSIN) is primarily focused on research examining the complex interplay among the brain, behavior and environment, utilizing multiple levels of analysis. These include behavioral, electrophysiological, pharmacological, cellular, genetic, molecular, and neural-network model approaches.

The topics covered in the journal include but not limited to: neuroimmune interactions and behavior; early-life experience and maternal care; cognitive development and aging; neural basis of vision and audition including multisensory and sensorimotor integration; large-scale oscillations and pharmacological mechanisms of spatial/ temporal processing; interval timing and temporal memory; neuralnetwork models of associative learning and sensory gating; sex differences in brain and behavior including numerical cognition and spatial navigation; nutrient and hormonal modulation of learning and memory across the lifespan; and the roles of the basal ganglia in goaldirected behavior and habit formation.

We would also like to include the topics from basic and translational neuroscience which aim to develop an understanding and treatment of major neurological diseases including Alzheimer's disease, stress related psychiatric disease such as depression and neuroendocrine diseases.

We welcome contributions that can demonstrate near-term practical usefulness, particularly contributions that take a multidisciplinary/ convergent approach because many real world problems are complex in nature.

JSIN provides an ideal forum for exchange of information on all of the above topics and more, in various formats: full length and letter length research papers, survey papers, work-in-progress reports on promising developments, case studies/best practice articles written by industry experts, and tutorials on up-and-coming technological breakthroughs. The journal's editorial board is strongly convinced this initiative will provide science-driven, peer-reviewed articles conforming to the strict international processes and editorial standards expected by the scientific community. JSIN is published six times a year. To ensure rapid dissemination of information, we aim at completing the review process of each paper within 2 months of initial submission.

JSIN is committed to publishing all manuscripts receiving a high or top priority recommendation during the review process, whereas those receiving medium priority will be considered for publication on a case-by-case basis. In addition, publication of manuscripts receiving the top priority will be fast tracked, that is, they will be published online within 1 week. This policy reflects my interest in quickly publishing all manuscripts judged to be the most impactful during the peer review process.

Further, we will periodically issue special calls for papers to modernize and strengthen areas of research and development showcased in Systems and Integrative Neuroscience and will be published as special issues.

I close this message by inviting everyone to submit their exciting research to JSIN. All papers receiving a high degree of enthusiasm in the peer-review process will find a home in JSIN. Therefore, we are committed to publishing all discoveries, methods, resources, and reviews that significantly advance the field of Neuroscience and its applications.

Once again I welcome you to this journal - your journal! With your support as authors, reviewers, and editors, I see very bright prospects for JSIN to serve science and the scientific community even better in the future. Ultimately, we will improve more lives and, consequently, our communities.
Copyright: (C2015 Perry G. This is an open-access article distributed under the terms of the Creative Commons Attribution License, which permits unrestricted use, distribution, and reproduction in any medium, provided the original author and source are credited.
Correspondence to: George Perry, E-mail: editor.jsin@oatext.com

Received: April 20, 2015; Accepted: April 21, 2015; Published: April 21, 2015 